\title{
Transporter-mediated ecdysteroid trafficking across cell membranes: A novel target for insect growth regulators
}

\author{
Naoki Okamoto ${ }^{1}$ and Naoki Yamanaka ${ }^{2, *}$ \\ ${ }^{1}$ Life Science Center for Survival Dynamics, Tsukuba Advanced Research Alliance, University of Tsukuba, Tsukuba, Ibaraki 305-8577, Japan \\ ${ }^{2}$ Department of Entomology, Institute for Integrative Genome Biology, University of California, Riverside, Riverside, CA 92521, USA
}

(Received October 31, 2020; Accepted December 16, 2020)

Ecdysteroids are a class of steroid hormones in arthropods that control molting and metamorphosis through interaction with intracellular nuclear receptors. In contrast to the extensive literature describing their biosynthetic pathways and signaling components, little has been known about how these hormones are traveling into and out of the cells through lipid bilayers of the cell membranes. Recently, a series of studies conducted in the fruit fly Drosophila melanogaster revealed that membrane transporters have critical functions in trafficking ecdysteroids across cell membranes, challenging the classical simple diffusion model of steroid hormone transport. Here we summarize recent advances in our understanding of membrane transporters involved in ecdysteroid signaling in Drosophila, with particular focus on Ecdysone Importer (Ecl) that is involved in ecdysteroid uptake in peripheral tissues. We then discuss the potential advantage of Ecl blockers as a novel pest management tool as compared to classical insect growth regulators.

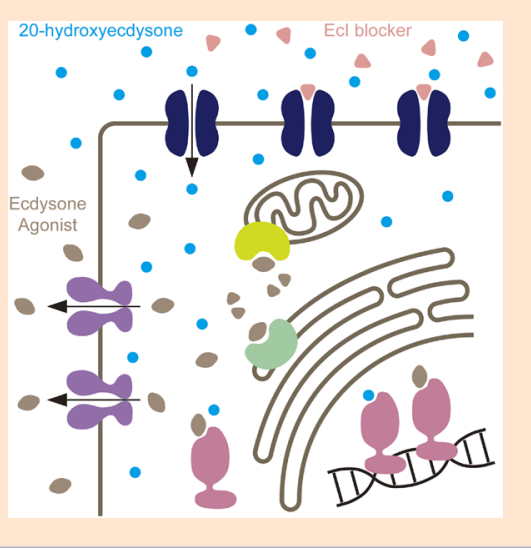

Keywords: ecdysone, ecdysteroid, EcR, Ecdysone Importer, IGR.

\section{Introduction}

As insects and other arthropods have a semi-rigid exoskeleton, they need to shed their old cuticle through a process called molting during growth. It has been long known that insect molting and metamorphosis are under endocrine control, ${ }^{1,2)}$ and the first "molting hormone" termed ecdysone was isolated in 1954. ${ }^{3)}$ Ecdysteroids, ecdysone and related compounds with molting hormone activities, are a group of steroid hormones that play a central role in the regulation of various developmental and physiological processes in arthropods including insects. ${ }^{4-6)} \mathrm{Be}-$ cause biosynthetic processes and signaling pathways of ecdysteroids have multiple levels of similarities with those of mam-

\footnotetext{
* To whom correspondence should be addressed.

E-mail: naoki.yamanaka@ucr.edu

Published online January 15, 2021
}

(c) BY-NC-ND $\odot$ Pesticide Science Society of Japan 2021. This is an open access article distributed under the Creative Commons Attribution-NonCommercial-NoDerivatives 4.0 International (CC BY-NC-ND 4.0) License (https://creativecommons.org/licenses/by-nc-nd/4.0/) malian steroid hormones, studies of insect ecdysteroids have significantly contributed to the advancement of the basic steroid hormone research, as exemplified by the first description of the genomic action of steroid hormones in dipteran flies. ${ }^{7)}$ More recently, application of the molecular genetic tools in the fruit fly Drosophila melanogaster has particularly contributed to the accumulation of knowledge about physiological processes that are both upstream and downstream of ecdysteroid signaling. ${ }^{8-10)}$ Moreover, besides basic biological studies, applied aspects of ecdysteroid signaling have also been extensively investigated in various insect species, in order to develop novel insect growth regulators (IGRs). ${ }^{11,12)}$ For example, diacylhydrazine (DAH)based non-steroidal ecdysone agonists that target the ecdysone receptor (EcR) have been developed and used as IGRs for over 30 years. ${ }^{13-15)}$

As steroid hormones are small lipophilic molecules, it has been widely accepted that they can simply diffuse across lipid bilayers of the plasma membranes. However, recent studies using Drosophila have challenged this simple diffusion model for ecdysteroids. Here we review recent findings of membrane transporter-mediated ecdysteroid trafficking across cell membranes and discuss the possibility of developing a novel class of IGRs 
that manipulate ecdysteroid transport for pest control.

\section{Vesicle-mediated ecdysteroid release from prothoracic gland cells}

During insect development, ecdysteroids are mainly synthesized from dietary sterols such as cholesterol or phytosterols in a specialized steroidal gland called the prothoracic gland (PG). ${ }^{9,16)}$ After transport of dietary sterols into the PG, biosynthesis of ecdysone (an immediate precursor of a biologically active ecdysteroid) is achieved through hydroxylation and oxidation steps mediated by a series of enzymes that are highly substrate-selective and predominantly expressed in the PG. ${ }^{16)}$ After secreted into the hemolymph, ecdysone is transported through the circulation to the peripheral tissues such as the gut and fat body, where it is converted to 20-hydroxyecdysone (20E; a biologically active ecdysteroid) by a cytochrome P450 monooxygenase named Shade. ${ }^{17)} 20 \mathrm{E}$ is released back into the hemolymph and re-enters its target cells, where it binds to the intracellular nuclear receptor complex composed of EcR and Ultraspiracle (USP). ${ }^{14,18,19)}$ The EcR/USP complex functions as a liganddependent transcription factor, which initiates the gene expression cascade that eventually leads to molting and metamorphosis. ${ }^{2,18,19)}$

In contrast to the extensive literature on ecdysteroid biosynthesis, little has been known about the mechanisms that regulate ecdysteroid secretion from the PG. This is due to the widely accepted notion that all steroid hormones are able to pass directly through lipid membranes because they are small lipophilic compounds. This is what is written in major biology textbooks today, and we generally believe that all steroid hormones can enter and exit cells freely across lipid bilayers by simple diffusion. Despite this widespread assumption, however, there are only a few reports where such simple diffusion of steroid hormones across cell membranes has been analyzed either in vitro or in silico. ${ }^{20,21)}$ The available evidence is, therefore, far too limited to be general-

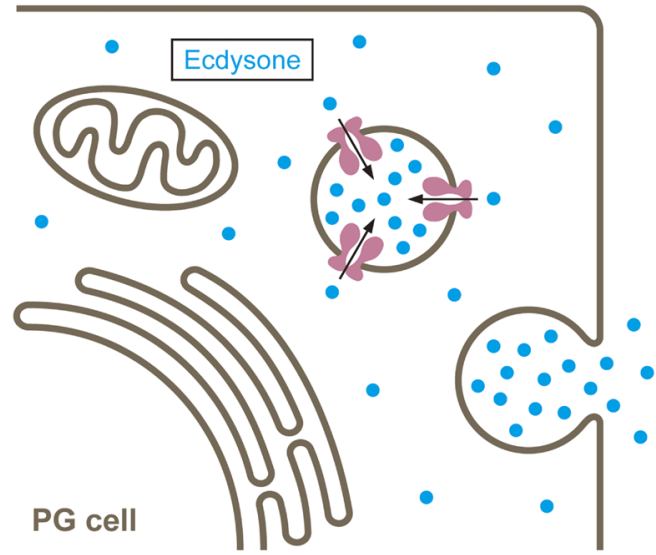

Fig. 1. Schematic diagram of ecdysone release from Drosophila PG cells. After its biosynthesis, ecdysone is incorporated into secretory vesicles by an $\mathrm{ABC}$ transporter called Atet (pink) and released into the hemolymph through exocytosis. ized to all steroid hormones.

In 2015, it was reported that in Drosophila, ecdysone is secreted into the hemolymph from the PG through a vesicle-mediated process, rather than simple diffusion. ${ }^{22)}$ Once synthesized in the PG, ecdysone is incorporated into secretory vesicles at high concentrations by an ATP-binding cassette (ABC) transporter called Atet and released into the hemolymph through exocytosis (Fig. 1). ${ }^{22)}$ Although this ecdysone transport happens against the concentration gradient through an ATP-dependent active transporter, the fact that ecdysone can be contained within vesicles suggested that ecdysteroids may not be able to freely traverse lipid bilayers. This opened up an intriguing possibility that the cellular uptake of ecdysteroids by peripheral tissues through lipid bilayers down the concentration gradient (termed facilitated diffusion) may also be controlled by a transporter-mediated process.

\section{Genetic screens identify a membrane transporter essential for ecdysteroid-dependent developmental transitions}

In 2018, two independent in vivo and in vitro genetic screens identified a membrane transporter that mediates cellular uptake of ecdysteroids in Drosophila. ${ }^{23)}$ The first screen conducted transgenic RNAi of $>500$ putative transporter-encoding genes to test for their requirement in ecdysteroid-dependent developmental events. The second screen utilized a recently developed pooled CRISPR screening approach in Drosophila S2 cells, which enables to generate cell pools of CRISPR mutants. ${ }^{24}$ Using this method, $>25,000$ sgRNAs targeting approximately 4,000 genes were screened for resistance in 20E-dependent cell cycle arrest. These two independent screens identified the same transporter-encoding gene, Oatp74D (CG7571), which was renamed as Ecdysone Importer $(E c I){ }^{23)}$

$E c I$ is required for 20E-dependent events in the salivary gland and fat body, and the EcI protein is localized on the plasma membrane in these tissues. Importantly, fat body-specific knockdown of EcI significantly reduced ecdysteroid levels within the fat body cells, indicating that EcI is essential for importing ecdysteroids into the cells. EcI null mutants developed normally as first instar larvae, but they stopped growing at the end of the first instar and failed to molt into the second instar. This indicates that EcI is required for the canonical function of $20 \mathrm{E}$ as the "molting hormone" in insects. It has been well known that the developmental arrest phenotype caused by defects in ecdysone production or secretion can be rescued by oral administration of 20E. ${ }^{25,26)}$ However, feeding $20 \mathrm{E}$ to the EcI mutants did not rescue their first instar arrest phenotype, further suggesting that $\mathrm{EcI}$ is required for $20 \mathrm{E}$ functions in vivo. In contrast, feeding a DAH-based non-steroidal ecdysone agonist chromafenozide (CF) to the EcI mutant larvae rescued the developmental arrest phenotype. Non-steroidal ecdysone agonists are a class of insecticides that mimic the action of $20 \mathrm{E}$ by binding directly to EcR, despite their structural differences with $20 \mathrm{E} .{ }^{14,15)}$ It is therefore likely that CF enters cells through a molecular machinery distinct from EcI (Fig. 2). Overall, these results indicate that EcI is 


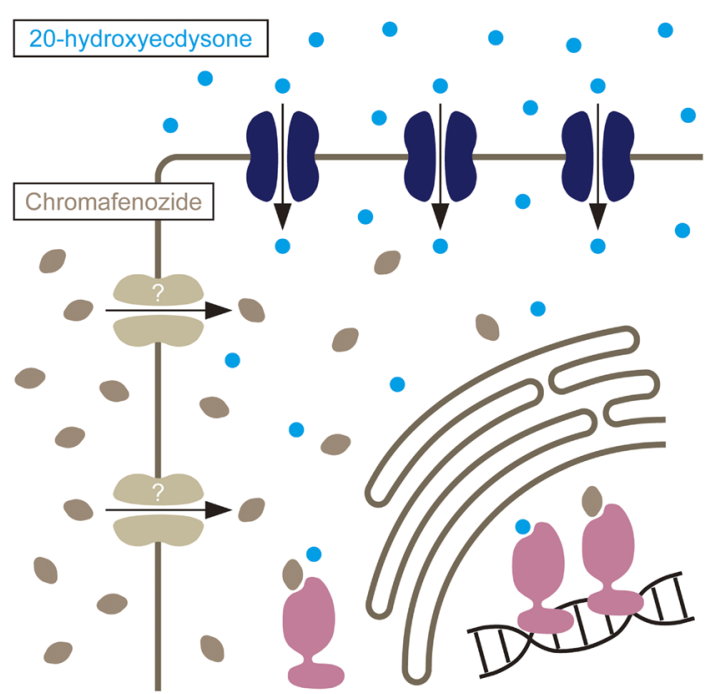

Fig. 2. Differential cellular entry mechanisms for $20 \mathrm{E}$ and CF. 20E is incorporated into its target cells through EcI (dark blue), whereas CF enters the cells through a distinct, unknown mechanism (brown). These agonists bind to EcR (pink) and induce gene expression.

a transporter that mediates ecdysteroid uptake in insects.

\section{Facilitated diffusion is necessary for cellular uptake of ecdysteroids}

The function of EcI was further analyzed using Drosophila S2 cells and mammalian HEK293 cells transfected with $E c R$ and a luciferase reporter construct with ecdysone response element $(E c R E)$. Knockdown of $E c I$ in S2 cells significantly reduced the response to 20E. Conversely, when EcI was overexpressed in S2 cells, their response to $20 \mathrm{E}$ was significantly increased. Importantly, neither EcI RNAi nor overexpression affected the cells' response to $\mathrm{CF}$, further suggesting that $\mathrm{CF}$ enters cells independently of EcI. Although the activity was significantly lower than that of 20E, the effect of ecdysone was also affected by EcI RNAi and overexpression, suggesting that both $20 \mathrm{E}$ and ecdysone are substrates for EcI. Indeed, overexpression of $E c I$ with Shade, the gene encoding a P450 enzyme that converts ecdysone to $20 \mathrm{E}$, significantly increased the efficiency of the ecdysoneto-20E conversion in S2 cells (unpublished result), suggesting that ecdysone likely enters peripheral tissues through EcI. Most importantly, heterologous expression of $E c I$ and $E c R$ in mammalian HEK293 cells makes them responsive to 20E, while expression of EcR alone does not. Collectively, these results demonstrate that EcI is both necessary and sufficient for inducing ecdysteroid-dependent gene expression in EcR-expressing animal cells. Ecdysteroid-inducible gene expression systems, where $E c R E$-driven gene expression is induced in a ligand-dependent manner, have been developed in EcR-expressing mammalian cells. ${ }^{27,28)}$ Interestingly, 20E is known to be incapable of inducing EcRE-driven gene expression in mammalian cells, but the reason why the endogenous ligand cannot activate EcR in mammalian cells has been elusive. ${ }^{29)}$ However, it is now clear that it is because mammalian cells do not express EcI. Considering that some other ecdysteroids have been used as potent ligands for the same gene expression systems, ${ }^{28)}$ it is likely that mammalian cells also possess high selectivity for the incorporation of different steroidal compounds. Altogether, this study clearly challenges the simple diffusion model of steroid hormone transport across cell membranes and instead indicates that a transporter-mediated, facilitated diffusion mechanism is required for ecdysteroid entry into target cells. ${ }^{23)}$

\section{Transporter-mediated ecdysteroid incorporation into the central nervous system}

In conjunction with extensive reorganization of the body structure during metamorphosis, insects need to transform the central nervous system (CNS) to adapt to adult-specific behaviors such as mating and flight. ${ }^{30,31)} 20 \mathrm{E}$ serves as a master regulator that evokes such transformation of the CNS including remodeling, differentiation, and programmed cell death in the CNS during metamorphosis. ${ }^{32-35)}$ Like mammals, the insect CNS is also physically separated from circulation by the blood-brain barrier (BBB), which acts as a highly selective gatekeeper to control the incorporation of molecules including hormones, to ensure normal neuronal functions. ${ }^{36-38)}$ Thus, in order to act on its target neurons, circulating 20E needs to enter the CNS by crossing the BBB. In Drosophila, the BBB consists of surface glial cell layers that cover the entire CNS. ${ }^{39-41)}$ Again, the conventional simple diffusion model does not indicate that such cell layers have a critical function in regulating steroid hormone entry into the CNS, whereas our transporter-mediated facilitated diffusion model gives those cell layers a critical gatekeeper role.

Importantly, immunohistochemical and gene expression analyses revealed that EcI is highly expressed in the BBB. ${ }^{42,43)}$ Indeed, EcI knockdown in the BBB severely impaired the CNS transformation, and the CNS retained its larval morphology even in the pupal stage. Furthermore, BBB-specific knockdown of EcI suppressed 20E signaling within the entire CNS and blocked 20E-mediated neuronal events such as remodeling, differentiation, and programmed cell death. Interestingly, BBBspecific knockdown of EcI caused a defect in wandering behavior, and the larvae mostly pupated on or in the food. Wandering behavior is an innate behavior induced by $20 \mathrm{E}$ through which larvae exit their food and prepare for pupation. ${ }^{44-46)}$ Furthermore, the majority of $E c I$ RNAi prepupae failed to perform head eversion during pupal ecdysis, another innate behavior known to be induced by $20 \mathrm{E} .{ }^{47)}$ These results, therefore, suggest that $\mathrm{EcI}$ in the $\mathrm{BBB}$ is required for circulating $20 \mathrm{E}$ to access neurons within the CNS that are responsible for 20E-mediated innate behaviors. The EcI function in the BBB was further investigated by using an ex vivo CNS culture system, where BBB-specific knockdown of EcI significantly reduced the response of the CNS to $20 \mathrm{E}$ in the culture media. This study thus demonstrates that EcI is required for ecdysteroid uptake into the CNS and provides important implications in controlling brain function by regulating ecdysteroid permeability across the BBB. ${ }^{43)}$ 


\section{Ecdysteroid transporters as novel targets for pest control}

As ecdysteroids play a pivotal role in regulating development and physiology in insects and other arthropods, components of the ecdysteroid signaling pathways have been selected as targets for the development of IGRs. ${ }^{11,12)}$ In particular, DAH-based non-steroidal ecdysone agonists targeting EcR have been used as effective IGRs for various insect species. Currently, five DAHbased IGRs are widely used as commercial insecticides, namely $\mathrm{CF}$, tebufenozide, methoxyfenozide, fufenozide, and halofenozide. $^{14,15)}$

When these effective IGRs that disrupt ecdysteroid signaling are already available, what is the advantage of developing new IGRs targeting novel players in ecdysteroid signaling, such as EcI? EcI is a member of the solute carrier organic anion transporter gene superfamily that encodes an organic anion transporting polypeptide (OATP) with 12 predicted transmembrane domains. ${ }^{23)}$ OATPs are widely present in the animal kingdom, and multiple chemicals with OATP-blocking activities have already been reported. ${ }^{48,49)}$ Importantly, our molecular phyloge-

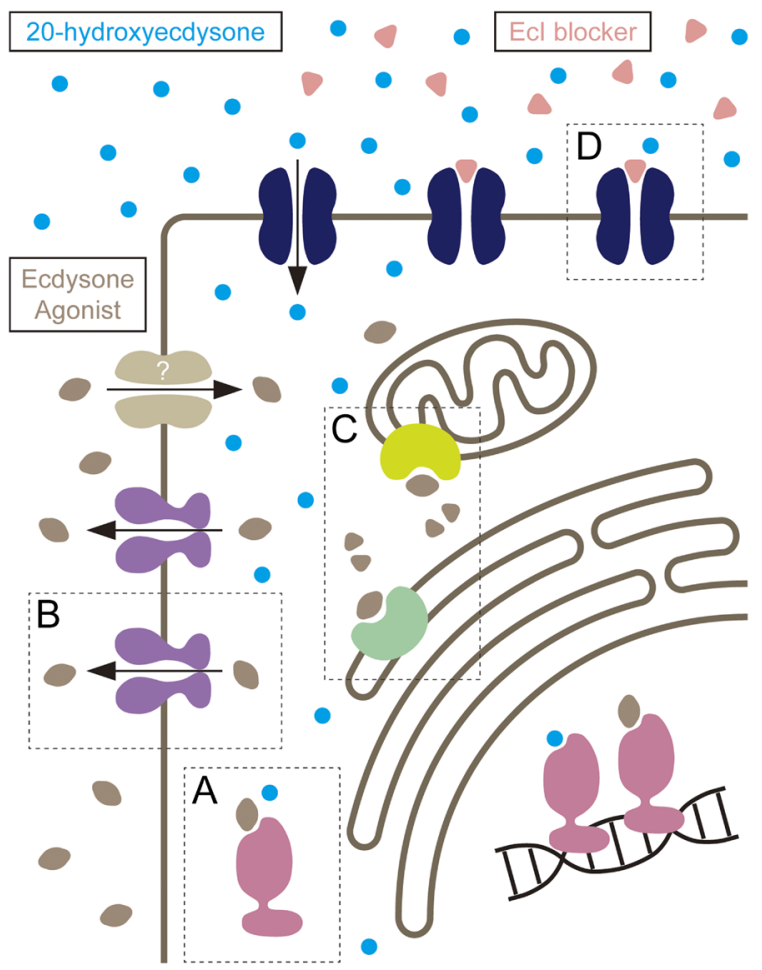

Fig. 3. Comparison of the modes-of-action of known ecdysone agonists and EcI blockers. 20E enters its target cells through EcI (dark blue) and binds to EcR (pink) to induce gene expression. Known ecdysone agonists also enter the cells to interact with EcR and disrupt ecdysteroid signaling (A), but this makes them susceptible to intracellular insecticide resistance machineries such as multidrug $\mathrm{ABC}$ transporters that pump out pesticides out of the cells (purple in B), or detoxification enzymes that break them down (green in C). In contrast, EcI blockers are expected to circumvent such resistance machinery as they can interact with EcI from extracellular space $(\mathrm{D})$. netic analysis revealed that a clear ortholog of $E c I$ is present only in arthropods that utilize ecdysteroids as the primary molting hormone. ${ }^{23)}$ It is, therefore, possible that arthropod-selective ecdysone antagonists can be developed by screening for potential EcI blockers. As compared to ecdysone agonists such as DAHbased IGRs, ecdysone antagonists have not been widely used as pest control reagents, and EcI blockers may broaden the target pest species that can be treated by IGRs.

Recently, resistance to DAH-based ecdysone agonists has been reported in field populations of several insect species. ${ }^{15)}$ This is partly because these IGRs need to enter the cells to interact with their target proteins, and therefore they become easy targets for intracellular detoxification enzymes such as cytochrome P450 monooxygenases, as well as multidrug ABC transporters that eliminate various pesticides out of the cells. ${ }^{50-53)}$ In this regard, EcI may become an ideal target for developing novel types of IGRs that are less likely to develop such insecticide resistance, as EcI is localized on the cell surface and its potential blockers can access their target protein directly from the hemolymph without entering the cytoplasm (Fig. 3). For the same reason, ecdysteroid efflux transporters in peripheral tissues, such as E23, ${ }^{54)}$ may also be an attractive target for developing similar types of IGRs.

\section{Concluding remarks}

Recent findings of ecdysteroid transporters in fruit flies critically challenged the simple diffusion model of ecdysteroid trafficking across cell membranes. Evidence is now accumulating in other systems that supports the facilitated diffusion mechanism of ecdysteroid transport, and the effort to identify effective EcI blockers is currently underway. As evidenced by the effective use of CF in the EcI research, identification of novel IGRs can not only provide novel methods of pest control, but also promote basic scientific studies. Considering that EcI is highly conserved in arthropods, EcI-blocking ecdysone antagonists are expected to promote our understanding of steroid hormone signaling in a diverse array of insects and beyond.

\section{References}

1) S. Kopeć: Studies on the necessity of the brain for the inception of insect metamorphosis. Biol. Bull. 42, 323-342 (1922).

2) J. W. Truman: The evolution of insect metamorphosis. Curr. Biol. 29, R1252-R1268 (2019).

3) A. Butenandt and P. Karlson: Über die isolierung eines metamorphose-hormons der insekten in kristallisierter form. Z. Naturforsch. B 9b, 389-391 (1954).

4) D. L. Mykles: Ecdysteroid metabolism in crustaceans. J. Steroid Biochem. Mol. Biol. 127, 196-203 (2011).

5) R. Lafont, C. Dauphin-Villemant, J. T. Warren and H. Rees: Ecdysteroid chemistry and biochemistry. In "Insect Endocrinology," ed. by L. I. Gilbert, Academic Press, London, pp. 106-176, 2012.

6) N. Yamanaka and N. Okamoto: Molecular functions of ecdysteroids in insects. In "Advances in Invertebrate (Neuro)Endocrinology," eds. by S. Saleuddin, A. B. Lange and I. Orchard, Apple Academic Press, New York, pp. 77-127, 2020. 
7) U. Clever and P. Karlson: Induktion von Puff-Veränderungen in Speicheldrüsenchromosomen von Chironomus tentans durch Ecdyson. Exp. Cell Res. 20, 623-626 (1960).

8) N. Yamanaka, K. F. Rewitz and M. B. O’Connor: Ecdysone control of developmental transitions: Lessons from Drosophila research. Annu. Rev. Entomol. 58, 497-516 (2013).

9) X. Pan, R. P. Connacher and M. B. O'Connor: Control of the insect metamorphic transition by ecdysteroid production and secretion. Curr. Opin. Insect Sci. 43, 11-20 (2021).

10) M. J. Texada, T. Koyama and K. Rewitz: Regulation of body size and growth control. Genetics 216, 269-313 (2020).

11) T. S. Dhadialla, A. Retnakaran and G. Smagghe: Insect growth and development disrupting insecticides. In "Comprehensive Molecular Insect Science Vol. 6: Control," eds. by L. I. Gilbert, K. Iatrou and S. G. Gill, Elsevier, Oxford, pp. 55-115, 2005.

12) M. Pener and T. S. Dhadialla: An overview of insect growth disruptors: Applied aspects. In "Advances in Insect Physiology, Vol 43," ed. by T. S. Dhadialla, Elsevier, London, pp. 1-162, 2012.

13) K. D. Wing, R. A. Slawecki and G. R. Carlson: RH 5849, a nonsteroidal ecdysone agonist: Effects on larval Lepidoptera. Science 241, 470-472 (1988)

14) Y. Nakagawa and V. C. Henrich: Arthropod nuclear receptors and their role in molting. FEBS J. 276, 6128-6157 (2009).

15) G. Smagghe, L. E. Gomez and T. S. Dhadialla: Bisacylhydrazine insecticides for selective pest control. In "Advances in Insect Physiology, Vol 43," ed. by T. S. Dhadialla, Elsevier, London, pp. 163-249, 2012.

16) R. Niwa and Y. S. Niwa: Enzymes for ecdysteroid biosynthesis: Their biological functions in insects and beyond. Biosci. Biotechnol. Biochem. 78, 1283-1292 (2014).

17) A. Petryk, J. T. Warren, G. Marqués, M. P. Jarcho, L. I. Gilbert, J. Kahler, J. P. Parvy, Y. Li, C. Dauphin-Villemant and M. B. O'Connor: Shade is the Drosophila P450 enzyme that mediates the hydroxylation of ecdysone to the steroid insect molting hormone 20-hydroxyecdysone. Proc. Natl. Acad. Sci. U.S.A. 100, 13773-13778 (2003).

18) L. M. Riddiford, P. Cherbas and J. W. Truman: Ecdysone receptors and their biological actions. Vitam. Horm. 60, 1-73 (2000).

19) K. King-Jones and C. S. Thummel: Nuclear receptors-A perspective from Drosophila. Nat. Rev. Genet. 6, 311-323 (2005).

20) S. Watanabe, T. Tani, S. Watanabe and M. Seno: Transport of steroid hormones facilitated by serum proteins. Biochim. Biophys. Acta 1073, 275-284 (1991)

21) I. Oren, S. J. Fleishman, A. Kessel and N. Ben-Tal: Free diffusion of steroid hormones across biomembranes: A simplex search with implicit solvent model calculations. Biophys. J. 87, 768-779 (2004).

22) N. Yamanaka, G. Marqués and M. B. O'Connor: Vesicle-mediated steroid hormone secretion in Drosophila melanogaster. Cell 163, 907919 (2015).

23) N. Okamoto, R. Viswanatha, R. Bittar, Z. Li, S. Haga-Yamanaka, N. Perrimon and N. Yamanaka: A membrane transporter is required for steroid hormone update in Drosophila. Dev. Cell 47, 294-305 (2018).

24) R. Viswanatha, Z. Li, Y. Hu and N. Perrimon: Pooled genome-wide CRISPR screening for basal and context-specific fitness gene essentiality in Drosophila cells. eLife 7, e36333 (2018).

25) H. Ono, K. F. Rewitz, T. Shinoda, K. Itoyama, A. Petryk, R. Rybczynski, M. Jarcho, J. T. Warren, G. Marques, M. J. Shimell, L. I. Gilbert and M. B. O'Connor: Spook and Spookier code for stage-specific components of the ecdysone biosynthetic pathway in Diptera. Dev. Biol. 298, 555-570 (2006).

26) T. Yoshiyama, T. Namiki, K. Mita, H. Kataoka and R. Niwa: Nev- erland is an evolutionally conserved Rieske-domain protein that is essential for ecdysone synthesis and insect growth. Development 133, 2565-2574 (2006).

27) K. S. Christopherson, M. R. Mark, V. Bajaj and P. J. Godowski: Ecdysteroid-dependent regulation of genes in mammalian cells by a Drosophila ecdysone receptor and chimeric transactivators. Proc. Natl. Acad. Sci. U.S.A. 89, 6314-6318 (1992).

28) E. Saez, M. C. Nelson, B. Eshelman, E. Banayo, A. Koder, G. J. Cho and R. M. Evans: Identification of ligands and coligands for the ecdysone-regulated gene switch. Proc. Natl. Acad. Sci. U.S.A. 97, 1451214517 (2000).

29) L. Dinan and R. Lafont: Effects and applications of arthropod steroid hormones (ecdysteroids) in mammals. J. Endocrinol. 191, 1-8 (2006).

30) J. W. Truman: Developmental neuroethology of insect metamorphosis. J. Neurobiol. 23, 1404-1422 (1992).

31) C. Consoulas, C. Duch, R. J. Bayline and R. B. Levine: Behavioral transformations during metamorphosis: Remodeling of neural and motor systems. Brain Res. Bull. 53, 571-583 (2000).

32) L. Veverytsa and D. W. Allan: Subtype-specific neuronal remodeling during Drosophila metamorphosis. Fly (Austin) 7, 78-86 (2013).

33) A. Boulanger and J. M. Dura: Nuclear receptors and Drosophila neuronal remodeling. Biochim. Biophys. Acta 1849, 187-195 (2015).

34) S. P. Yaniv and O. Schuldiner: A fly's view of neuronal remodeling. Wiley Interdiscip. Rev. Dev. Biol. 5, 618-635 (2016).

35) M. H. Syed, B. Mark and C. Q. Doe: Playing well with others: Extrinsic cues regulate neural progenitor temporal identity to generate neuronal diversity. Trends Genet. 33, 933-942 (2017).

36) M. K. DeSalvo, N. Mayer, F. Mayer and R. J. Bainton: Physiologic and anatomic characterization of the brain surface glia barrier of Drosophila. Glia 59, 1322-1340 (2011).

37) S. Limmer, A. Weiler, A. Volkenhoff, F. Babatz and C. Klämbt: The Drosophila blood-brain barrier: Development and function of a glial endothelium. Front. Neurosci. 8, 365 (2014).

38) S. J. Hindle and R. J. Bainton: Barrier mechanisms in the Drosophila blood-brain barrier. Front. Neurosci. 8, 414 (2014).

39) S. D. Carlson, J. L. Juang, S. L. Hilgers and M. B. Garment: Blood barriers of the insect. Annu. Rev. Entomol. 45, 151-174 (2000).

40) T. Schwabe, R. J. Bainton, R. D. Fetter, U. Heberlein and U. Gaul: GPCR signaling is required for blood-brain barrier formation in Drosophila. Cell 123, 133-144 (2005).

41) T. Stork, D. Engelen, A. Krudewig, M. Silies, R. J. Bainton and C. Klämbt: Organization and function of the blood-brain barrier in Drosophila. J. Neurosci. 28, 587-597 (2008).

42) M. K. DeSalvo, S. J. Hindle, Z. M. Rusan, S. Orng, M. Eddison, K. Halliwill and R. J. Bainton: The Drosophila surface glia transcriptome: Evolutionary conserved blood-brain barrier processes. Front. Neurosci. 8, 346 (2014).

43) N. Okamoto and N. Yamanaka: Steroid hormone entry into the brain requires a membrane transporter in Drosophila. Curr. Biol. 30, 359366 (2020).

44) O. S. Dominick and J. W. Truman: The physiology of wandering behaviour in Manduca sexta. II. The endocrine control of wandering behaviour. J. Exp. Biol. 117, 45-68 (1985).

45) B. L. Hall and C. S. Thummel: The RXR homolog Ultraspiracle is an essential component of the Drosophila ecdysone receptor. Development 125, 4709-4717 (1998).

46) M. B. Davis, G. E. Carney, A. E. Robertson and M. Bender: Phenotypic analysis of $E c R-A$ mutants suggests that $\mathrm{EcR}$ isoforms have unique functions during Drosophila development. Dev. Biol. 282, 385-396 (2005). 
47) D. Žitňan and M. E. Adams: Neuroendocrine regulation of ecdysis. In "Insect Endocrinology," ed. by L. I. Gilbert, Academic Press, London, pp. 253-309, 2012.

48) A. Kalliokoski and M. Niemi: Impact of OATP transporters on pharmacokinetics. Br. J. Pharmacol. 158, 693-705 (2009).

49) B. Hagenbuch and B. Stieger: The SLCO (former SLC21) superfamily of transporters. Mol. Aspects Med. 34, 396-412 (2013).

50) G. Smagghe, T. S. Dhadialla, S. Derycke, L. Tirry and D. Degheele: Action of the ecdysteroid agonist tebufenozide in susceptible and artificially selected beet armyworm. Pestic. Sci. 54, 27-34 (1998).

51) G. Smagghe, B. Carton, L. Decombel and L. Tirry: Significance of absorption, oxidation, and binding to toxicity of four ecdysone agonists in multi-resistant cotton leafworm. Arch. Insect Biochem. Physiol. 46, 127-139 (2001).
52) A. Retnakaran, I. Gelbic, M. Sundaram, W. Tomkins, T. Ladd, M. Primavera, Q. Feng, B. Arif, R. Palli and P. Krell: Mode of action of the ecdysone agonist tebufenozide (RH-5992), and an exclusion mechanism to explain resistance to it. Pest Manag. Sci. 57, 951-957 (2001).

53) M. Uchibori-Asano, A. Jouraku, T. Uchiyama, K. Yokoi, G. Akiduki, Y. Suetsugu, T. Kobayashi, A. Ozawa, S. Minami, C. Ishizuka, Y. Nakagawa, T. Daimon and T. Shinoda: Genome-wide identification of Tebufenozide resistant genes in the smaller tea tortrix, Adoxophyes honmai (Lepidoptera: Tortricidae). Sci. Rep. 9, 4203 (2019).

54) T. Hock, T. Cottrill, J. Keegan and D. Garza: The E23 early gene of Drosophila encodes an ecdysone-inducible ATP-binding cassette transporter capable of repressing ecdysone-mediated gene activation. Proc. Natl. Acad. Sci. U.S.A. 97, 9519-9524 (2000). 